Research Article

\title{
Circulating MicroRNA Expression, Vitamin D, and Hypercortisolism as Predictors of Osteoporosis in Elderly Postmenopausal Women
}

\author{
Hadeel A. Al-Rawaf, ${ }^{1}$ Ahmad H. Alghadir, ${ }^{2}$ and Sami A. Gabr $\mathbb{D}^{2}$ \\ ${ }^{1}$ Department of Clinical Laboratory Sciences, College of Applied Medical Sciences, King Saud University, Riyadh, Saudi Arabia \\ ${ }^{2}$ Department of Rehabilitation Sciences, College of Applied Medical Sciences, King Saud University, Riyadh, Saudi Arabia
}

Correspondence should be addressed to Sami A. Gabr; dr.samigabr@gmail.com

Received 16 May 2021; Revised 20 November 2021; Accepted 30 November 2021; Published 13 December 2021

Academic Editor: Gaetano Isola

Copyright (c) 2021 Hadeel A. Al-Rawaf et al. This is an open access article distributed under the Creative Commons Attribution License, which permits unrestricted use, distribution, and reproduction in any medium, provided the original work is properly cited.

\begin{abstract}
Background. MicroRNAs (miRNA) identified as critical molecular regulators for bone development, function, and modeling/ remodeling process and could be predictable for osteoporotic fractures in postmenopausal elderly women. Aim. The potential diagnostic role of circulating miRNAs, miR-148a and miR-122-5p, in the pathogenesis of osteoporosis and its association with bone markers, hypercortisolism, and vitamin D deficiency were explored in postmenopausal elderly women with osteoporosis. Methods. A total of 120 elderly women aged 50-80 years old were recruited in this study, of which only 100 eligible women with amenorrhea of at least 12 consecutive months or surgical menopause participated in this study. Based upon bone mineral density (BMD) measurements, the participants were classified according into two groups: normal $(n=45 ; T$ score of $\geq-1.0)$ and osteoporosis $(n=55 ; T$ score: $\leq-2.5)$. Circulating miRNAs, miR-148a and miR-122-5p, were estimated by real-time RT-PCR analysis. In addition, bone markers, hypercortisolism, and vitamin D deficiency were colorimetrically and ELISA immune assay estimated. The potential role of miR-148a, miR-122-5p, cortisol, and vitamin $\mathrm{D}$ in the diagnosis of osteoporosis was predicted using the analysis of the respective area under the receiver operating characteristic curve (AUCROC). Results. The expressed level of miR-148a significantly increased and miR-122-5p significantly decreased in the serum of osteoporotic patients compared to healthy controls. In addition, a significant increase in the levels of cortisol, s-BAP, and CTx and significant decrease in the levels of T-BMD, the levels of OC, and s-Ca were also identified. All parameters significantly correlated with fracture risk parameters; BMD, and $T$ score lumbar spine (L2-L4). Thus, the data showed AUC cut off values (miR-148a; 0.876, miR-122-5p; 0.761) were best evaluated for clinical diagnosis of patients with osteoporosis and that AUC cut off values of 0.748 for cortisol and 0.635 for vitamin D were the best cut off values, respectively, reported for the prediction of osteoporosis clinical diagnosis. Conclusion. In this study, expressed miRNAs miR-148a and miR-122-5p and changes in the levels of both cortisol and vitamin D status are significantly associated with bone loss or osteoporosis. Thus, circulation miRNAs alone or in combination with cortisol and vitamin D status might be considered predictable biomarkers in the diagnosis or the pathogenesis of osteoporosis in elderly postmenopausal women; however, more studies are recommended.
\end{abstract}

\section{Introduction}

Bones are the most supporting component comprising the musculoskeletal system. It serves to support the body, assisting in movement, and protects vital organs as well as participates in producing blood cells, storing nutrients and minerals of the human bodies [1].
In normal biological processes, bone tissue is continuously controlled by hemostatic physiological modeled and remodeled processes to maintain a healthy bone matrix and to adapt to the change in environmental factors [1-3]. Thus, any disorder in these mechanisms significantly results in a reduction in bone mass with an increased risk for fractures with aging particularly, in postmenopausal women, impacting bone health $[4,5]$. 
Osteoporosis is one of the most metabolic bone disease characterized by an imbalance in skeletal remodeling, resulting in an increase in the activity of osteoclast and/or a decrease in the numbers of osteoblasts. These hemostatic changes significantly lead to a decrease in bone strength, mass, bone quality, bone density, and bone geometry with lower bone mineral density (BMD) of $\leq 2.5$ standard deviations below peak bone mass which significantly increases the susceptibility to severe bone fracture especially around age 30 [6-9]. BMD considered one of the most important measurements for predicting osteoporotic fractures among older ages $[10,11]$. However, other several physiological factors, such as hormones, vitamin D deficiency, cytokines, and mechanical stimulation, significantly affect resorption and formation of bone markers and mediate the complex regulation processes of bone homeostasis [12-25].These factors could interfere with other factors such as exercise, age, sex, and diet with the pathogenesis and severity of osteoporosis [26].

In addition, many studies reported that proinflammatory cytokines and their related genes such as galectin, NLRP3, and IL-6 have a significant pivotal role in the destruction of bones and severity of osteoporosis. More genes like interleukin 6 (IL-6), vitamin D receptor (VDR), estrogen receptor (ESR), and calcitonin receptor (CTR) as well others were suggested to be associated with osteoporosis and bone density [27-34]. Previous clinical studies found that IL-6 and other inflammatory cytokines accelerate bone destruction and osteoporosis via upregulation of the expression and the signal transduction of RANKL and on osteoblasts, increasing vertebral fractures and the severity of bone loss or osteoporosis [35-38].

Pathophysiological hypotheses reported that proinflammatory cytokines like IL-6 along with matrix GLA protein and growth factors are associated with bone turnover and in turn have a role in bone loss or osteoporosis in patients with ischemic cardiovascular diseases [39]. Additionally, NLRP3 inflammasome was activated as inflammatory promoters in patients with osteoporosis which was significantly suppressed by melatonin use in patients with postmenopausal osteoporosis [40, 41].

As general, galectins as carbohydrate-binding proteins interfere in many physiological processes particularly inflammatory and immune response showed to be associated with activation of other proinflammatory markers such as NLRP3 inflammasome and aid in the progression of osteoporosis $[42,43]$. NLRP3 and galectins showed to be significantly elevated in the serum and saliva of patients with periodontitis, confirming the association of with a multifactorial inflammatory disease $[33,34]$. In addition, clinical studies reported that asymptomatic hypercortisolism is associated with osteoporotic fractures and that subclinical hypercortisolism (SH) is among the causes with bone fragility particularly those with slightly reduced BMD and vertebral fractures [44].

In addition, non-coding short RNA molecules (18-25 nucleotides) showed to be associated with many diseases [26-28] including the bone loss or osteoporosis [29-32].

Several environmental and genetic factors significantly interfere with the incidence and the pathogenesis of osteopo- rosis [14]. Epigenetic mechanisms including microRNA expression is shown to be associated as posttranscriptional regulators of gene expression $[45,46]$. The prominent role of miRNAs was demonstrated in many physiological processes proceeding in the pathogenesis of a number of diseases [47]. Remarkable stability, protection from degradation, tissue specificity, and easy accessibility of circulating miRNAs molecules support its use as biomarkers for early diagnosis for diseases and monitoring of treatment [48-52]. Previous studies reported that extracellular miRNAs represent as a cellular waste product and might be required in intercellular communication $[48,49]$. In bone formation, miRNAs are identified to be linked with the differentiation, proliferation, and function of both osteoblasts and osteoclasts [53]. Also, bone homeostasis was regulated by the miRNAs via direct or indirect impact on the expression of various transcription factors and cytokines required in the process of bone resorption and bone formation [53-55]. In addition, the functional role of microRNAs in bone regulation suggests their vital role as biomarkers for pathological changes associated with osteoporosis [56]. This support the role of miRNAs in many bone diseases which is not fully studied [53, 56-64]; however, their biological role in the pathogenesis of bone and its association with other physiological parameters like hypercortisolism and vitamin D deficiency are still unclear. From the previously mentioned studies [14,45-55], it was reported that in postmenopausal patients, more physiological parameters like hypercortisolism, vitamin D deficiency, and circulating miRNAs were associated with osteoporotic fractures. However, in postmenopausal women with osteoporosis, there are little or no intercorrelations were fully studied between expressed circulating miRNAs with hypercortisolism, vitamin D deficiency, and physiological bone markers. Thus, in this study, the potential diagnostic role of circulating miRNAs, miR148a and miR-122-5p, in the pathogenesis of osteoporosis and its association with bone markers, hypercortisolism, and vitamin $\mathrm{D}$ deficiency were explored in postmenopausal elderly women with osteoporosis.

\section{Materials and Methods}

2.1. Subjects. A total of 120 elderly women aged $50-80$ years old were recruited in this study, of which only 100 eligible women with amenorrhea of at least 12 consecutive months or surgical menopause participated in this study. Based upon bone mineral density (BMD) measurements, the participants were classified according into two groups: normal $(n=45 ; T$ score of $\geq-1.0)$ and osteoporosis $(n=55$; $T$ score: $\leq-2.5)$. Study exclusion criteria included women with severe disease complications such as diabetes mellitus, chronic liver diseases, rheumatoid arthritis or collagen, endocrine disorders which affect bone mass, hyperthyroidism, spondylitis, systemic lupus erythematosus, connective tissue disease, metabolic and endocrine diseases, bone tumours, or who received corticosteroid therapy, hormone replacement therapy, stress hormones, or the miRNA measurements, and bisphosphonates treatment that could interfere with BMD measurements. In addition, patients with low physical 
activity, prevalent fractures, or with parental history of bone disorders were excluded either from the current study.

The study protocol was reviewed according to the ethical guidelines of the 1975 Declaration of Helsinki and approved by the ethical committee of Rehabilitation Research Chair (RRC), King Saud University, Kingdom of Saudi Arabia, under file number (ID: RRC-2019-096), and signed informed consent forms were received from all subjects prior data collection.

2.2. Diet and Duration of Sun Exposure. During the entire period of data collection, all participants were informed not to change their normal eating habits and to record accurately the amount, type of food, and fluid consumed. Then, dietary information was obtained from food diaries of each participant or by extensive dietary interviews and significantly referred according to reference dietary intakes previously reported $[65,66]$. In addition, daily sun exposure was estimated by the number of weeks spent in the sun by each student and divided by seven to estimate the average number of minutes per day the participants; normal and osteoporosis were exposed to sunlight $[65,66]$.

2.3. Blood Sampling. Following an overnight fast, blood samples were obtained from all participants at 9:00-11:30 a.m. to avoid probable diurnal influence. The proposed time for blood collection was selected for control of the circadian hormonal range, as previously reported in the procedures of other studies [67].

2.4. Assessment of BMD Measurements. All participants were subjected to perform BMD measurements of the lumbar spine, total hip, femoral neck and $1 / 3$ radius by using dualenergy X-ray absorptiometry (Discovery, Hologic, Waltham, MA) as previously reported [68]. Osteoporosis was significantly defined according to the criteria of World Health Organisation (WHO) [69-71]. According to these diagnostic criteria, subjects were classified as osteoporosis (DXA T scores of $\leq-2.5$ standard deviation $(\mathrm{SD})$ ), osteopenia ( $T$ scores of $-1.0 \mathrm{SD}$ to $-2.5 \mathrm{SD}$ ), and normal [ $T$ scores of $\geq-1 \mathrm{SD}]$, respectively [68-71].

2.5. Assessment of Bone Markers. Serum levels of calcium, bone alkaline phosphatase (sBAP), osteocalcin (OC), and C-telopeptide (CTx) were estimated in all participants as previously reported $(63,67,68,69$, and 16$)$. Serum calcium and BAP concentrations were estimated by a Cobas Integra colorimetric analyzer with the aid of commercially available immunoassay ELISA kits (Hoffman-LaRoche Ltd., Basel, Switzerland) for calcium and the immunoenzymetric assay kits (MicroVue BAP, Quidel Corporation, San Diego, CA) for serum BAP concentrations [69-71].

In addition, serum levels of OC and CTx were determined using enzyme immunoassay analysis [72, 73]. The levels were measured with ELISA kits (the MicroVue Osteocalcin enzyme immunoassay, Quidel Corporation, San Diego, CA) for OC and enzyme immunoassay kits (Serum CrossLaps One Step ELISA, Osteometer BioTech, Herlev, Denmark)) for serum CTx, respectively $[72,73]$.
2.6. Assessment of Vitamin 25(OH)D and Cortisol. Serum concentrations of vitamin $25(\mathrm{OH}) \mathrm{D}$ and cortisol were estimated in all subjects as previously mentioned in the literature $[74,75]$. In this experiment, immunoassay ELISA kits (IDS, Tyne \& Wear, UK) and (ELISA, Diagnostics Biochem. Canada Inc.) were used to estimate the levels of vitamin $25(\mathrm{OH}) \mathrm{D}$ and cortisol concentrations, respectively, in the serum of all participants.

\subsection{Real-Time RT-PCR Analysis of Circulating miRNAs and Apoptotic Genes}

2.7.1. Extraction of RNA and Synthesis of $c D N A$. For each participant, the miRNease isolation kit (Qiagen, Hilden, Germany) was used to extract total RNA from serum samples. A reverse-transcription polymerase chain reaction (RTPCR) was applied to analyze total RNA in all serum samples. Then, a complementary DNA (c-DNA) was generated using reverse transcription miScriptII RT kits (Qiagen), and the levels of miRNAs were evaluated by optical density $[76,77]$.

2.7.2. Real-Time RT-PCR Analysis. The primers of circulating miRNAs miR-148a and miR-122-5p (Applied Biosystems, Foster City, CA, U.S.A.) were used to screen the expression of miRNAs in the plasma of all participants by using a quantitative real-time RT-PCR [78]. The average copy number of the resultant PCR components was normalized according to the GAPDH gene which is used as an internal housekeeping gene $[79,80]$. In the PCR process, templets of respective cDNA were subjected to four thermal phases: primary denaturation phase (I) (at $94^{\circ} \mathrm{C}$ for 2 minutes), denaturation phase (II) (at $94^{\circ} \mathrm{C}$ for 30 seconds), annealing phase (III) (at $59^{\circ} \mathrm{C}$ for 30 seconds), and amplification phase (IV) (at $72^{\circ} \mathrm{C}$ for 30 seconds). The PCR phases (II to IV) proceed for 45 cycles and all reactions were measured in a triplicated manner [81].

2.7.3. Statistical Analysis. Power calculations of the selected sample size of 100 subjects showed to give an estimated power of $96 \%$ and a significance level of 0.05 with an expected frequency of $9.6 \%$.

An SPSS statistical program (SPSS, IBM Statistics V.17) was used to analyze all data produced in this study. The data of continuous variables are expressed as mean \pm SD. The frequency differences between the groups were analyzed by using a nonparametric test (Mann-Whitney-Wilcoxon test) and the $\chi^{2}$ test, respectively. In all groups, two independent sample $t$-tests were used for comparison between the studied variables such as osteoporosis (dependent variable), expression levels of miRNAs, bone markers, vitamin $25(\mathrm{OH}) \mathrm{D}$, and serum cortisol levels (independent variables). In addition, multiple stepwise regressions and Pearson's correlations analysis were used to estimate the associations between bone loss (osteoporosis) and the studied independent variables in control subjects and in postmenopausal women with osteoporosis. The susceptibility and sensitivity of cortisol, vitamin D, miR-148a, and miR-122-5p for osteoporosis diagnosis at baseline expression were determined using the area under the receiver operating characteristic 
(ROC) curve as previously reported [81]. All tests were twotailed; because of multiple assessments, results were only considered statistically significant at a value of $p<0.05$.

\section{Results}

In this study, a total of 100 eligible women with amenorrhea of at least 12 consecutive months participated in this study. Only $55 \%$ of the study population reported osteoporosis with $T$ score $(\leq-2.5)$ and $45 \%$ of the population had normal $T$ score $(\geq-1.0)$, respectively (Table 1$)$. Fracture risk parameters, T-BMD, BMD-femoral neck, BMD-total hip, BMDlumbar spine, BMD $1 / 3$, and $T$ score, were significantly decreased $(p=0.001)$ in osteoporotic women compared to healthy controls, whereas BMD ( $z$ and $T$ scores) at FN and at LS were significantly different $(p=0.001)$ (Table 1$)$.

In addition, deficient in dietary vitamin $\mathrm{D}$ and $\mathrm{Ca}$ intake were significantly reported in osteoporotic women $(p=0.001)$ compared to healthy controls.

In this study, the effect of vitamin D and cortisol levels on the bone loss was estimated in healthy and osteoporotic women (Figure 1). A significant decrease $(p=0.001)$ in the levels of vitamin D (a) and increase $(p=0.001)$ in the levels of cortisol (b) were reported in osteoporotic postmenopausal women compared to healthy controls. In addition, a significant decrease $(p=0.001)$ in the T-BMD, the levels of OC and $s-\mathrm{Ca}$, and increased levels of s-BAP and CTx $(p=0.001)$, respectively, in osteoporotic postmenopausal women compared to healthy controls (Figures 1(c)-1(e)).

Molecular changes associated with osteoporosis in postmenopausal women were also studied (Figure 2). MicroRNAs' differential expression profile was estimated in healthy control and in postmenopausal women with osteoporosis. The results showed that the relative expression of miR-148a significantly increased $(p=0.001)$, and miR-122$5 \mathrm{p}$ significantly reduced $(p=0.001)$ in postmenopausal women with osteoporosis compared to healthy controls.

In women with osteoporosis, the association of fracture risk parameters; T-BMD and T score lumbar spine (L2-L4) with cofounders were evaluated (Table 2). Both T-BMD and $T$ score correlated negatively with the change in the levels of vitamin-25 (OH)D, cortisol, OC, and s-Ca and positively correlated with CTx and s-BAP, respectively, as shown in Table 2.

In addition, the cellular expression microRNAs; miR$148 \mathrm{a}$ and $\mathrm{miR}-122-5 \mathrm{p}$ were significantly associated $(p=0.001)$ with the levels of vitamin-25 (OH)D, cortisol, and bone markers: OC, CTx, s-Ca, and s-BAP. The expression of miR-148a and miR-122-5p correlated positively with OC, CTx, s-Ca, and s-BAP and negatively with BMD, and $T$ score lumbar spine (L2-L4), the levels of vitamin-25 (OH)D, cortisol, respectively, as shown in Table 3.

Diagnostic values for cortisol, vitamin D, miR-148a, and miR-122-5p in clinical samples for osteoporosis patients were determined using the area under the receiver operating characteristic (ROC) curve (Table 4). There were acceptable diagnostic values for the levels of miR-148a $(\mathrm{AUC}=0.876$, $p=0.01)$ and miR-122-5p (AUC $=0.761, p=0.05)$ in clinical samples for osteoporosis patients (Table 4). The sensitivity and specificity for miR-148a in clinical samples were
TABle 1: The demographics and baseline bone parameters of the participants.

\begin{tabular}{|c|c|c|}
\hline Variables & $\begin{array}{l}\text { Normal } \\
\text { group }\end{array}$ & $\begin{array}{c}\text { Osteoporosis } \\
\text { group }\end{array}$ \\
\hline$N$ & $45(45 \%)$ & $55(55 \%)$ \\
\hline Age (years) & $62.6 \pm 4.1$ & $63.9 \pm 3.2$ \\
\hline BMI $\left(\mathrm{kg} / \mathrm{m}^{2}\right)$ & $24.9 \pm 3.4$ & $22.5 \pm 2.6^{\mathrm{b}}$ \\
\hline Waist $(\mathrm{cm})$ & $96.8 \pm 9.3$ & $79.2 \pm 6.3^{\mathrm{b}}$ \\
\hline Hips $(\mathrm{cm})$ & $93.7 \pm 6.4$ & $89.8 \pm 8.3^{\mathrm{b}}$ \\
\hline WHR & $1.034 \pm 0.13$ & $0.882 \pm 0.09^{b}$ \\
\hline Years of menopause & $7.5 \pm 1.5$ & $12.5 \pm 2.0^{\mathrm{b}}$ \\
\hline BMD femoral neck (FN; $\mathrm{g} / \mathrm{cm}^{2}$ ) & $0.751 \pm 0.61$ & $0.610 \pm 0.39^{\mathrm{b}}$ \\
\hline$T$ score for FN & $0.38 \pm 0.56$ & $-2.6 \pm 0.75^{\mathrm{b}}$ \\
\hline$Z$ score for FN & $1.3 \pm 0.46$ & $-1.42 \pm 0.82^{b}$ \\
\hline BMD lumbar spine (LS; $\mathrm{g} / \mathrm{cm}^{2}$ ) & $0.985 \pm 0.86$ & $0.692 \pm 0.58^{\mathrm{a}}$ \\
\hline$T$ score for LS (L2-L4) & $0.71 \pm 1.3$ & $-2.8 \pm 0.98^{\mathrm{b}}$ \\
\hline$Z$ score for LS & $0.48 \pm 1.1$ & $-3.1 \pm 0.98^{\mathrm{b}}$ \\
\hline BMD total hip $\left(\mathrm{g} / \mathrm{cm}^{2}\right)$ & $0.865 \pm 0.812$ & $0.715 \pm 0.59^{\mathrm{b}}$ \\
\hline BMD $1 / 3$ radius $\left(\mathrm{g} / \mathrm{cm}^{2}\right)$ & $0.68 \pm 0.23$ & $0.51 \pm 0.48^{\mathrm{b}}$ \\
\hline Total BMD $\left(\mathrm{g} / \mathrm{cm}^{2}\right)$ & $0.91 \pm 0.08$ & $0.65 \pm 0.09^{\mathrm{b}}$ \\
\hline Sun exposure (min/d) & $125 \pm 15.8$ & $80 \pm 12.7^{\mathrm{b}}$ \\
\hline Dietary vitamin D intake (IU/d) & $215 \pm 22.5$ & $118 \pm 8.3^{\mathrm{b}}$ \\
\hline Dietary Ca intake (mg/d) & $1350 \pm 148$ & $789 \pm 31.5^{\mathrm{b}}$ \\
\hline
\end{tabular}

Values are expressed as mean $\pm \mathrm{SD}$; significance at $p<0.05$. ${ }^{\mathrm{a}} p<0.01$, ${ }^{\mathrm{b}} p<0.001$. BMD: bone mineral density; BMI: body mass index; WHR: waist-to-hip ratio.

91.5\% and $89.3 \%$ compared to that of miR-122-5p, $82.9 \%$ and $86.7 \%$, respectively. In addition, there was a much stronger diagnostic value for osteoporosis (AUC $=0.820, P=$ 0.001 ) reported when both microRNAs combined together than either separately (Table 4).

Similarly, there were acceptable diagnostic values for the levels of cortisol $(\mathrm{AUC}=0.748, p=0.01)$ and vitamin $\mathrm{D}$ (AUC $=0.635, p=0.01$ ) in clinical samples for osteoporosis patients (Table 4). The sensitivity and specificity for cortisol in clinical samples were $89.8 \%$ and $85.2 \%$ compared to that of vitamin $\mathrm{D} 85.8 \%$ and $84.6 \%$, respectively. In addition, there was a much stronger diagnostic value for osteoporosis (AUC $=0.789, p=0.001)$ reported when both cortisol and vitamin $\mathrm{D}$ combined together than either separately (Table 4). The results suggest that both microRNAs, miR122-5p and miR-4516, along with cortisol, and vitamin D status could be used together to increase diagnostic value for osteoporosis.

\section{Discussion}

Osteoporosis comprises a major public health problem characterized by a reduction in bone mass and deterioration in bone microarchitecture with lower bone mineral density 


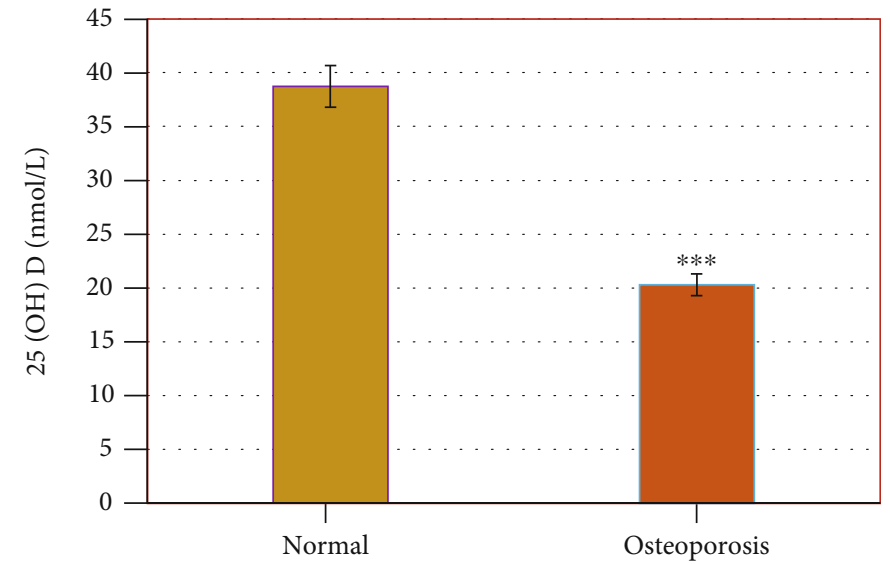

(a)

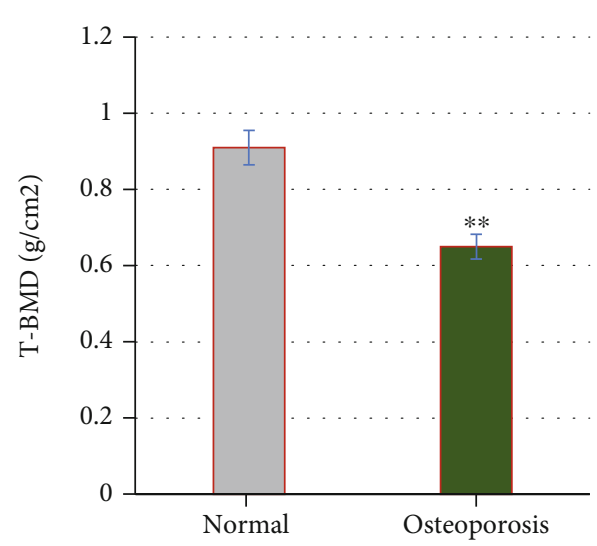

(c)

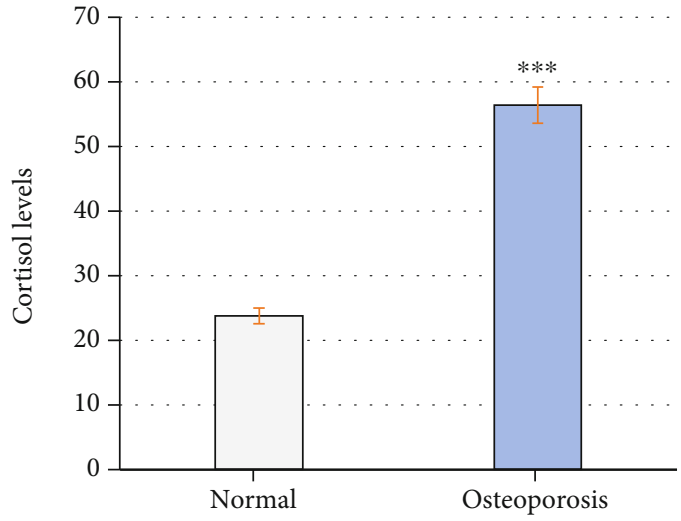

(b)

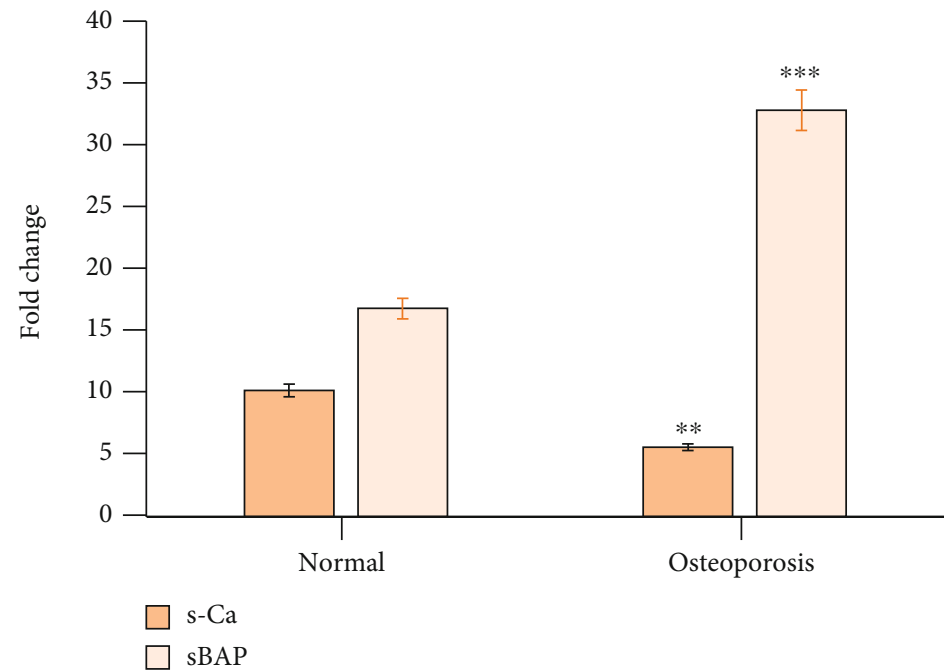

(d)

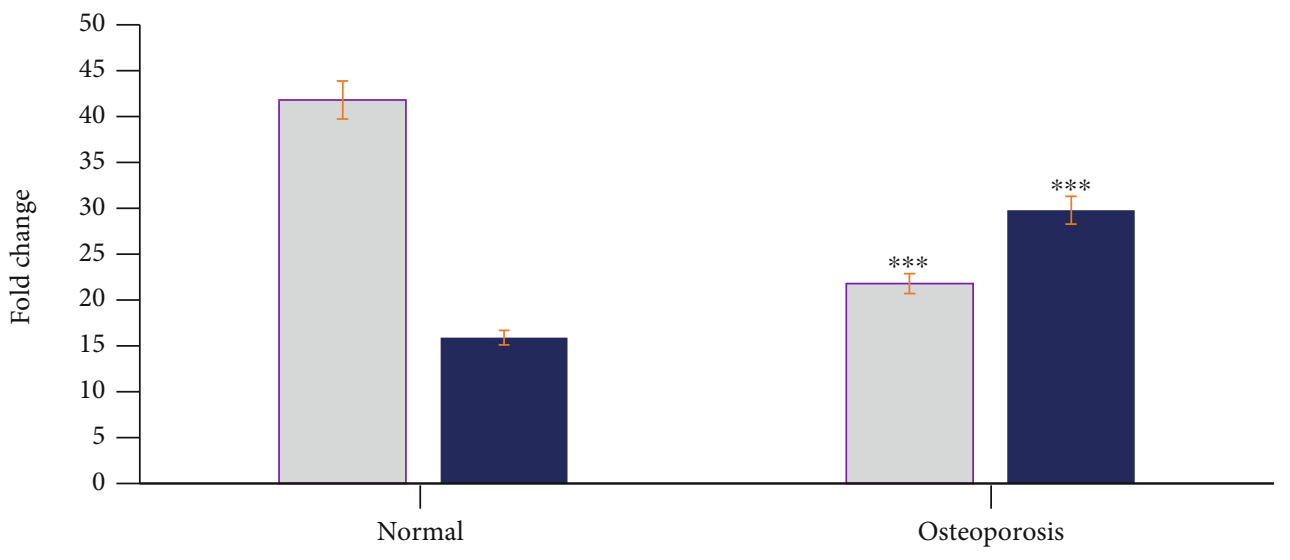

$\mathrm{OC}(\mathrm{ng} / \mathrm{ml})$

CTx (ng/ml)

(e)

FIGURE 1: Changes in vitamin D and cortisol $(\mathrm{a}, \mathrm{b})$ total bone mineral density and bone markers; OC, CTx; sBAP and s-Ca the levels (c-e) in healthy control and in postmenopausal women with osteoporosis. The results showed significant decrease $(p=0.001)$ in the levels of vitamin $\mathrm{D}$ (a) and increase $(p=0.001)$ in the levels of cortisol (b) in osteoporotic postmenopausal women compared to healthy controls. In addition, significant decrease $(p=0.001)$ in the T-BMD, the levels of OC, s-Ca, and increased levels of sBAP, CTx $(p=0.001)$, respectively, in osteoporotic postmenopausal women compared to healthy controls $(\mathrm{c}-\mathrm{e}) .{ }^{* *} p \leq 0.01,{ }^{* * *} p \leq 0.001$. OC: osteocalcin; CTx: collagen type I c-telopeptide; sBAP: serum bone alkaline phosphatase; s-Ca: serum calcium. 


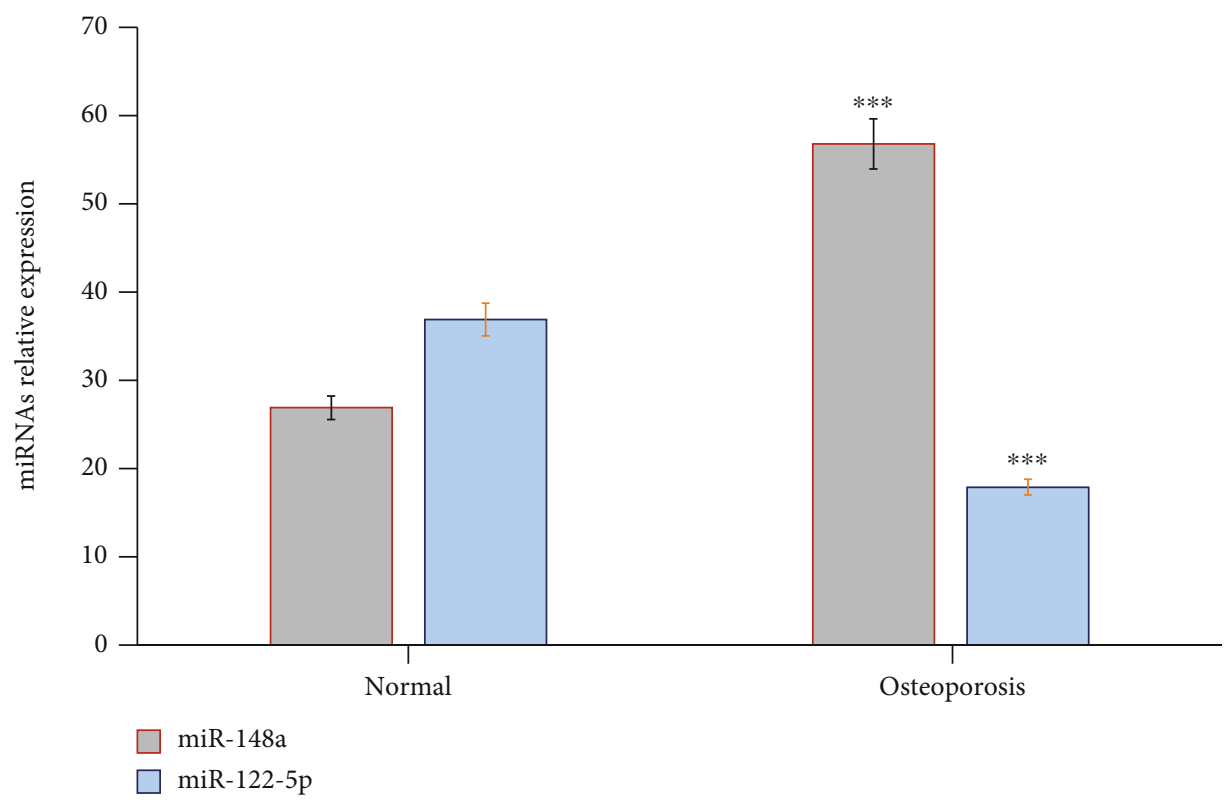

FIGURE 2: MicroRNAs' differential expression profile in healthy control and in postmenopausal women with osteoporosis. The results showed that the relative expression of miR-148a significantly increased $(p=0.001)$ and miR-122-5p significantly reduced $(p=0.001)$ in postmenopausal women with osteoporosis compared to healthy controls. ${ }^{\mathrm{a}} p \leq 0.01,{ }^{\mathrm{b}} p \leq 0.001$.

TABLE 2: Correlations between bone markers, cortisol, vitamin D levels, miRNAs, and fracture risk parameters in postmenopausal women with osteoporosis.

\begin{tabular}{|c|c|c|}
\hline \multirow[b]{2}{*}{$\begin{array}{l}\text { Studied } \\
\text { parameters }\end{array}$} & \multicolumn{2}{|c|}{ Fracture parameters } \\
\hline & $\begin{array}{l}\text { Total BMD } \\
\left(\mathrm{g} / \mathrm{cm}^{2}\right)\end{array}$ & $\begin{array}{c}T \text { score lumbar } \\
\text { spine (L2-L4) }\end{array}$ \\
\hline OC (ng/ml) & $-0.27^{\mathrm{a}}$ & $-0.36^{\mathrm{b}}$ \\
\hline CTx (ng/ml) & $0.23^{\mathrm{a}}$ & $0.14^{\mathrm{b}}$ \\
\hline $\mathrm{s}-\mathrm{Ca}$ & $-0.24^{\mathrm{a}}$ & $-0.26^{\mathrm{b}}$ \\
\hline s-BAP & $0.38^{\mathrm{a}}$ & $0.42^{\mathrm{b}}$ \\
\hline $\begin{array}{l}\text { Vitamin-25 } \\
(\mathrm{OH}) \mathrm{D}(\mathrm{nmol} / \mathrm{l})\end{array}$ & $-0.31^{\mathrm{a}}$ & $-0.42^{\mathrm{b}}$ \\
\hline Cortisol & $-0.18^{\mathrm{a}}$ & $-0.21^{b}$ \\
\hline
\end{tabular}

Data are presented as Pearson's $(R)$ coefficients adjusting for variables identified as cofounders in univariate analyses. Significance at $p<0.05$. ${ }^{\mathrm{a}} p<0.01,{ }^{\mathrm{b}} p<0.001$. BMD: bone mineral density; OC: osteocalcin; CTx: collagen type I c-telopeptide; sBAP: serum bone alkaline phosphatase; s-Ca: serum calcium.

(BMD) values ( $T$ score; 2.5 s.d or more) at the spine or hip regions which significantly below the average calculated values for healthy young women [82].

In this study, osteoporosis was estimated in 55\% $(n=55)$ of the study population with $T$ score $(\leq-2.5)$. Fracture risk parameters: T-BMD, BMD-femoral neck, BMD-total hip, BMD-lumbar spine, BMD $1 / 3$, and $T$ score were significantly decreased $(p=0.001)$ in osteoporotic women compared to healthy controls ( $T$ score; $\geq-1.0$ ).

An increase in osteoporosis was detected among postmenopausal women which significantly depend mainly upon the type of clinical techniques for BMD measurement [81-83]. In these studies, although, there was significant variability in detecting osteoporosis between used techniques
TABle 3: Correlations between bone markers, cortisol, vitamin D levels, and miRNAs in postmenopausal women with osteoporosis.

\begin{tabular}{lcc}
\hline \multirow{2}{*}{ Studied parameters } & \multicolumn{2}{c}{ miRNA expression } \\
& miR-148a & miR-122-5p \\
\hline OC (ng/ml) & $0.76^{\mathrm{b}}$ & $0.64^{\mathrm{b}}$ \\
CTx (ng/ml) & $0.42^{\mathrm{b}}$ & $0.31^{\mathrm{b}}$ \\
s-Ca & $0.58^{\mathrm{a}}$ & $0.69^{\mathrm{b}}$ \\
s-BAP & $0.18^{\mathrm{a}}$ & $0.23^{\mathrm{b}}$ \\
Cortisol & $-0.37^{\mathrm{b}}$ & $-0.49^{\mathrm{b}}$ \\
Vitamin-25 (OH)D (nmol/l) & $-0.17^{\mathrm{a}}$ & $-0.39^{\mathrm{b}}$ \\
Total BMD (g/cm $\left.{ }^{2}\right)$ & $-0.51^{\mathrm{a}}$ & $-0.24^{\mathrm{a}}$ \\
T score lumbar spine (L2-L4) & $-0.63^{\mathrm{b}}$ & $-0.27^{\mathrm{b}}$ \\
\hline
\end{tabular}

Data are presented as Pearson's $(R)$ coefficients adjusting for variables identified as cofounders in univariate analyses. Significance at $p<0.05$. ${ }^{\mathrm{a}} p<0.01,{ }^{\mathrm{b}} p<0.001$. OC: osteocalcin; CTx: collagen type I c-telopeptide; sBAP: serum bone alkaline phosphatase; $s-C a:$ serum calcium.

such as QCT and DXA; osteoporosis still present in higher values among postmenopausal women.

Our results were significantly supported by an abnormal change in the levels of both resorption and formationrelated bone markers. In osteoporotic postmenopausal women, the levels of OC and s-Ca significantly decreased and the levels of s-BAP and CTx significantly increased compared to healthy control women.

Previously, it was suggested that bone turnover markers such as bone resorption and bone formation markers can be used alone or in association with other bone parameters for assessing bone loss or osteoporosis in aged menopause women $[84,85]$.

After menopause, the role of endogenous glucocorticoids and deficient in vitamin $\mathrm{D}$ in physiological bone loss remain 
TABle 4: Diagnostic value of mi-R-148a, miR-122-5p, cortisol, and vitamin D for osteoporosis $(n=55)$.

\begin{tabular}{|c|c|c|c|}
\hline \multirow{2}{*}{ miRNAs } & \multicolumn{3}{|c|}{ AUC for diagnosis of osteoporosis (Ops) } \\
\hline & Area $(95 \% \mathrm{CI})$ & Sensitivity & Specificity \\
\hline mi-R-122-5p & $0.761^{\mathrm{a}}(0.686-0.896)$ & 82.9 & 86.7 \\
\hline miR-148a & $0.876^{\mathrm{b}}(0.798-0.965)$ & 91.5 & 89.3 \\
\hline$m i-R-148 a+m i R-122-5 p$ & $0.820^{c}(0.720-0.910)$ & 94.2 & 89.6 \\
\hline Cortisol & $0.748^{\mathrm{b}}(0.650-0.875)$ & 89.8 & 85.2 \\
\hline Vitamin D & $0.635^{\mathrm{b}}(0.580-0.785)$ & 85.8 & 84.6 \\
\hline Cortisol+vitamin D. & $0.789^{\mathrm{c}}(0.650-0.896)$ & 90.8 & 87.3 \\
\hline
\end{tabular}

AUC: area under the curve; CI: confidence interval. ${ }^{\mathrm{a}} p<0.05 .{ }^{\mathrm{b}} p<0.01 .{ }^{\mathrm{c}} p<0.001$.

to be explored. Subclinical hypercortisolism $[86,87]$ and lower vitamin levels were reported among older patients with established osteoporosis $[88,89]$.

Thus, in the current study, a significant decrease $(p=0.001)$ in the levels of vitamin D with an increase $(p=0.001)$ in the levels of cortisol was reported in osteoporotic postmenopausal women compared to healthy controls.

Our results like others suggest that the physiological changes in cortisol levels significantly associated with impairment of bone mass and bone quality in physiological menopause. Thus, postulate that the parameters of the hypothalamic-pituitary-adrenal (HPA) axis function may contribute to postmenopausal bone health [89-92]. The prevalence of subclinical hypercortisolism was reported in about $10 \%$ of outpatients with established osteoporosis. Thus, the presence of hypercortisolism has to be taken into account when evaluating patients with unexplainable established osteoporosis [93]. In addition, adequate levels of serum vitamin $\mathrm{D}$ are required to protect against bone fracture $[94,95]$ and that the complications of vitamin D deficiency particularly the vitamin $\mathrm{D}$ status and mutations in the vitamin $\mathrm{D}$ receptor markedly contribute to bone health among elderly women [94, 96-98].

In this study, compared to controls, T-BMD and $T$ score lumbar spine (L2-L4) as fracture risk parameters in osteoporotic patients correlated negatively with deficient vitamin-25 $(\mathrm{OH}) \mathrm{D}$, higher cortisol levels, and lower OC and s-Ca levels and positively correlated with higher levels of both CTx and $s$-BAP. It was reported previously that the deficiency in vitamin D levels was related to several factors particularly disturbance in the synthesis of physiological hormones which affects the production of the active forms of vitamin D [99-101].

Similarly, 25(OH)D and bone turnover markers (BTMs) shown to be associated with the incidence of hip fractures in older adults [102-110].Thus, a deficiency in serum $25(\mathrm{OH}) \mathrm{D}$ levels considered one of the most risk factors for the incidence of hip fractures in older women [102].

In addition, several studies investigated the unrecognized risk factors for bone loss [13, 110-113] especially cortisol. Like our results, others reported that excessive production of cortisol can lead to diminished bone metabolism and altered bone architecture [114], whereas a dysregulation of the hypothalamic-pituitary-adrenal (HPA) system significantly leads to secretions of cortisol in higher concentrations in the serum cortisol of osteoporotic patients which in turn is associated with a decrease in bone formation and increase in bone resorption with an overall decline in bone mineral content $[13,103-115]$.

Additionally, molecular-based studies represent microRNAs (miRNA) as critical molecular regulators that are involved in the bone remodeling processes, whereas several miRNAs showed to participate in several mechanisms especially, bone development, function, modeling, and remodeling process [101-104]. In addition, it plays a potential role in the characterization of the phenotype of bone cells (osteocytes, osteoblasts, and osteoclasts) $[105,106]$. Thus, miRNAs showed to be involved in bone diseases [107].

In this study, the potential roles of miRNAs as molecular markers in the pathogenesis and early diagnosis of osteoporosis were estimated in osteoporotic patients by using a quantitative real-time RT-PCR. The results showed a significant increase in baseline serum miR-148a and a decrease in the levels of miR-122-5p in osteoporotic patients compared to healthy controls, respectively. The cellular expression of microRNAs miR-148a and miR-122-5p significantly correlated with risk parameters: T-BMD and $T$ score lumbar spine (L2-L4), the levels of vitamin-25 (OH)D, cortisol, and bone markers OC, CTx, s-Ca, and s-BAP. The expression of miR-148a and miR-122-5p correlated positively with OC, CTx, s-Ca, and s-BAP and negatively with T-BMD and $T$ score lumbar spine (L2-L4), vitamin-25 (OH)D, and cortisol, respectively.

Recently, the increased levels of miR-148a significantly correlated with the main parameters of bone metabolism: bone mineral density (BMD) total body (TB) values, calcium, osteocalcin, bone alkaline phosphatase (sBAP), and vitamin-25 (OH)D (VitD) [116]. These data significantly assigned the potential role of miR-148a in both bone metabolism alterations and remodeling.

Also, previous studies reported the increase of miR-148a in plasma of osteoporotic (OP) postmenopausal women [117] and in sera of those with reported hip fractures [118]. Thus, the circulating expression of miR-148a signature suggested a potential for bone remodeling and could be considered as a potential biomarker of bone diseases $[13,110-113]$.

MicroRNAs are expressed in cells and significantly reported as critical molecular regulators capable of modifying the expression of genes at a posttranscriptional level $[13,114]$. This proceeds via inhibiting the translation of particular mRNAs or inducing specific mRNA degradation 
[115-117]. Consistent with our results, hsa-miR-122-5p was significantly downregulated in serum samples from osteoporosis patients compared to healthy nonosteoporosis subjects. These confirmed that the expressed microRNAs; miR-148a, miR-122-5p were associated with a fragility fracture and the low bone mineral density measured by T-BMD, and T-score lumbar spine (L2-L4 in osteoporosis patients $[118,119]$.

In the current study, ROC curve analysis showed that miR-148a, miR-122-5p along with both cortisol and vitamin $25(\mathrm{OH}) \mathrm{D}$ levels could be identified as strong diagnostic osteoporotic biomarkers. The results are in line with previously reported studies that confirmed cellular expressional changes in the levels of both miR-148a, miR-122-5p in elderly women with osteoporosis [13, 113-119]. The expression of miR-122-5p is differentially down-regulated and miR-148a is differentially upregulated in the serum of osteoporotic elderly women $[13,112-120]$. In addition, cortisol significantly increased [97, 98, 113, 115-117] and vitamin $25(\mathrm{OH}) \mathrm{D}$ levels significantly reduced $[103,106,107,111]$ in osteoporotic women and clinically associated with bone fractures and low bone mineral density in osteoporosis patients.

Finally, although BMD measurement is gold standard for diagnosis of osteoporosis/osteopenia, the results obtained signify circulating miR-148a, miR-122-5p along with cortisol, and vitamin $\mathrm{D}$ status as potential diagnostic biomarkers for osteoporosis. Thus, these markers might be useful in cases where DXA analysis might be severe or unsuitable for application such as pregnancy.

\section{Conclusion}

In this study, expressed miRNAs miR-148a and miR-122-5p and changes in the levels of both cortisol and vitamin D status significantly associated with bone loss or osteoporosis. Thus, circulation miRNAs alone or in combination with cortisol and vitamin D status might be considered as predictable biomarkers in diagnosis or the pathogenesis of osteoporosis in elderly postmenopausal women, however more studies are recommended.

\section{Data Availability}

All data generated or analyzed during this study are presented in the manuscript. Please contact the corresponding author for access to data presented in this study.

\section{Conflicts of Interest}

The authors declare that they have no competing interests.

\section{Acknowledgments}

The authors are grateful to the Researchers Supporting Project number (RSP-2021/382), King Saud University, Riyadh, Saudi Arabia for funding this research.

\section{References}

[1] P. M. Wippert, M. Rector, G. Kuhn, and K. Wuertz-Kozak, "Stress and alterations in bones: an interdisciplinary perspective," Frontiers in Endocrinology, vol. 8, no. 8, p. 96, 2017.

[2] L. J. Raggatt and N. C. Partridge, "Cellular and Molecular Mechanisms of Bone Remodeling*," The Journal of Biological Chemistry, vol. 285, no. 33, pp. 25103-25108, 2010.

[3] J. Klein-Nulend, R. G. Bacabac, and A. D. Bakker, "Mechanical loading and how it affects bone cells: the role of the osteocyte cytoskeleton in maintaining our skeleton," European Cells \& Materials, vol. 24, pp. 278-291, 2012.

[4] J. A. Cauley, "Public health impact of osteoporosis," Journals of Gerontology Series A: Biomedical Sciences and Medical Sciences, vol. 68, no. 10, pp. 1243-1251, 2013.

[5] S. R. Cummings and L. J. Melton, "Epidemiology and outcomes of osteoporotic fractures," Lancet, vol. 359, no. 9319, pp. 1761-1767, 2002.

[6] J. A. Kanis, "Assessment of fracture risk and its application to screening for postmenopausal osteoporosis: synopsis of a WHO report," Osteoporosis International, vol. 4, no. 6, pp. 368-381, 1994.

[7] A. H. Simpson and I. R. Murray, "Main differences in osteoporotic fracture models: which should I use?," Injury, vol. 47, Suppl 1, pp. S15-S20, 2016.

[8] A. Kleyer and G. Schett, "Arthritis and bone loss," Current Opinion in Rheumatology, vol. 26, no. 1, pp. 80-84, 2014.

[9] G. Haugeberg, T. Uhlig, J. A. Falch, J. I. Halse, and T. K. Kvien, "Bone mineral density and frequency of osteoporosis in female patients with rheumatoid arthritis: results from 394 patients in the Oslo County Rheumatoid Arthritis register," Arthritis and Rheumatism, vol. 43, no. 3, pp. 522-530, 2000.

[10] S. W. Wade, C. Strader, L. A. Fitzpatrick, M. S. Anthony, and C. D. O'Malley, "Estimating prevalence of osteoporosis: examples from industrialized countries," Archives of Osteoporosis, vol. 9, no. 1, p. 182, 2014.

[11] World Health Organization, "Assessment of fracture risk and its application to screening for postmenopausal osteoporosis," Technical report series 843, WHO, Geneva, 1994.

[12] J. Chen, K. Li, Q. Pang et al., "Identification of suitable reference gene and biomarkers of serum miRNAs for osteoporosis," Scientific Reports, vol. 6, no. 1, article 36347, 2016.

[13] L. Gennari, S. Bianciardi, and D. Merlotti, "MicroRNAs in bone diseases," Osteoporosis International, vol. 28, no. 4, pp. 1191-1213, 2017.

[14] G. Hendrickx, E. Boudin, and W. Van Hul, "A look behind the scenes: the risk and pathogenesis of primary osteoporosis," Nature Reviews Rheumatology, vol. 11, no. 8, pp. 462474, 2015.

[15] G. Wheater, M. Elshahaly, S. P. Tuck, H. K. Datta, and J. M. van Laar, "The clinical utility of bone marker measurements in osteoporosis," Journal of Translational Medicine, vol. 11, no. 1, p. 201, 2013.

[16] H. K. Datta, W. F. Ng, J. A. Walker, S. P. Tuck, and S. S. Varanasi, "The cell biology of bone metabolism," Journal of Clinical Pathology, vol. 61, no. 5, pp. 577-587, 2008.

[17] J. J. Carey, A. A. Licata, and M. F. Delaney, "Biochemical markers of bone turnover," Clinical Reviews in Bone and Mineral Metabolism, vol. 4, no. 3, pp. 197-212, 2006. 
[18] A. Leibbrandt and J. M. Penninger, "RANK/RANKL: regulators of immune responses and bone physiology," Annals of the New York Academy of Sciences, vol. 1143, no. 1, pp. 123-150, 2008.

[19] M. Zaidi, "Skeletal remodeling in health and disease," Nature Medicine, vol. 13, no. 7, pp. 791-801, 2007.

[20] P. Torricelli, M. Fini, G. Giavaresi, and R. Giardino, "Human osteoblast cultures from osteoporotic and healthy bone: biochemical markers and cytokine expression in basal conditions and in response to 1,25 (OH)2D3," Artificial Cells, Blood Substitutes, and Immobilization Biotechnology, vol. 30, no. 3, pp. 219-227, 2002.

[21] G. Osella, M. Ventura, A. Ardito et al., "Cortisol secretion, bone health, and bone loss: a cross-sectional and prospective study in normal non-osteoporotic women in the early postmenopausal period," European Journal of Endocrinology, vol. 166, no. 5, pp. 855-860, 2012.

[22] I. Chiodini, M. L. Mascia, S. Muscarella et al., "Subclinical hypercortisolism among outpatients referred for osteoporosis," Annals of Internal Medicine, vol. 147, no. 8, pp. 541-548, 2007.

[23] A. G. Turner, P. H. Anderson, and H. A. Morris, "Vitamin D and bone health," Scandinavian Journal of Clinical and Laboratory Investigation, vol. 243, pp. 65-72, 2012.

[24] C. Capatina, M. Carsote, A. Caragheorgheopol, C. Poiana, and M. Berteanu, "Vitamin d deficiency in postmenopausal women-biological correlates," Maedica, vol. 9, no. 4, pp. 316-322, 2014.

[25] H. A. Morris, "Vitamin D activities for health outcomes," Annals of Laboratory Medicine, vol. 34, no. 3, pp. 181-186, 2014.

[26] L. M. Havill, M. C. Mahaney, T. L Binkley, and B. L Specker, "Effects of genes, sex, age, and activity on BMC, bone size, and areal and volumetric BMD," Journal of Bone and Mineral Research, vol. 22, no. 5, pp. 737-746, 2007.

[27] B. Chen and H.-Z. Li, "Association of IL-6 174G/C (rs1800795) and 572C/G (rs1800796) polymorphisms with risk of osteoporosis: a meta-analysis," BMC Musculoskeletal Disorders, vol. 21, no. 1, p. 330, 2020.

[28] W. Xie, L. Ji, T. Zhao, and P. Gao, "Identification of transcriptional factors and key genes in primary osteoporosis by DNA microarray," Medical Science Monitor, vol. 21, pp. 13331344, 2015.

[29] G. R. Clark and E. L. Duncan, "The genetics of osteoporosis," British Medical Bulletin, vol. 113, no. 1, pp. 73-81, 2015.

[30] E. Bandrés, I. Pombo, M. González-Huarriz, A. Rebollo, G. López, and J. García-Foncillas, "Association between bone mineral density and polymorphisms of the VDR, ERalpha, COL1A1 and CTR genes in Spanish postmenopausal women," Journal of Endocrinological Investigation, vol. 28, no. 6, pp. 312-321, 2005.

[31] Y. M. Mosaad, E. M. Hammad, Z. Fawzy et al., "Vitamin D receptor gene polymorphism as possible risk factor in rheumatoid arthritis and rheumatoid related osteoporosis," Human Immunology, vol. 75, no. 5, pp. 452-461, 2014.

[32] A. Nordström, P. Gerdhem, H. Brändström et al., "Interleukin-6 promoter polymorphism is associated with bone quality assessed by calcaneus ultrasound and previous fractures in a cohort of 75-year-old women," Osteoporosis International, vol. 15, no. 10, pp. 820-826, 2004.

[33] G. Isola, A. Polizzi, A. Alibrandi, R. C. Williams, and A. Lo Giudice, "Analysis of galectin-3 levels as a source of coronary heart disease risk during periodontitis," Journal of Periodontal Research, vol. 56, no. 3, pp. 597-605, 2021.

[34] G. Isola, A. Polizzi, S. Santonocito, A. Alibrandi, and R. C. Williams, "Periodontitis activates the NLRP3 inflammasome in serum and saliva," Journal of Periodontology, 2021.

[35] T. Kishimoto, M. Hibi, M. Murakami, M. Narazaki, M. Saito, and T. Taga, "The molecular biology of interleukin 6 and its receptor,” Ciba Foundation Symposium, vol. 167, pp. 5-16, 1992.

[36] Y. O. Ishimi, C. H. Miyaura, C. H. Jin et al., "IL-6 is produced by osteoblasts and induces bone resorption," Journal of Immunology, vol. 145, no. 10, pp. 3297-3303, 1990.

[37] A. J. Littlewood, J. Russell, G. R. Harvey, D. E. Hughes, R. G. G. Russell, and M. Gowen, "The modulation of the expression of IL-6 and its receptor in human osteoblasts in vitro," Endocrinology, vol. 129, no. 3, pp. 1513-1520, 1991.

[38] S. H. Ralston, "Do genetic markers aid in risk assessment?," Osteoporosis International, vol. 8, Suppl 1, pp. S37-S42, 1998.

[39] J. R. Arron and Y. Choi, "Bone versus immune system," Nature, vol. 408, no. 6812, pp. 535-536, 2000.

[40] M. Laroche, V. Pécourneau, H. Blain et al., "Osteoporosis and ischemic cardiovascular disease," Joint Bone Spine, vol. 84, no. 4, pp. 427-432, 2017.

[41] L. Xu, L. Zhang, Z. Wang et al., "Melatonin suppresses estrogen deficiency-induced osteoporosis and promotes osteoblastogenesis by inactivating the NLRP3 inflammasome," Calcified Tissue International, vol. 103, no. 4, pp. 400-410, 2018.

[42] L. Johannes, R. Jacob, and H. Leffler, "Galectins at a glance," Journal of Cell Science, vol. 131, no. 9, article jcs208884, 2018.

[43] A. Arsenijevic, B. Stojanovic, J. Milovanovic, D. Arsenijevic, N. Arsenijevic, and M. Milovanovic, "Galectin-3 in inflammasome activation and primary biliary cholangitis development," International Journal of Molecular Sciences, vol. 21, no. 14, p. 5097, 2020.

[44] A. Lasco, A. Catalano, A. Pilato, G. Basile, A. Mallamace, and M. Atteritano, "Subclinical hypercortisol-assessment of bone fragility: experience of single osteoporosis center in Sicily," European Review for Medical and Pharmacological Sciences, vol. 18, no. 3, pp. 352-358, 2014.

[45] M. Sun, X. Zhou, L. Chen et al., "The regulatory roles of microRNas in bone remodeling and perspectives as biomarkers in osteoporosis," BioMed Research International, vol. 15, no. 8, pp. 509-524, 2016.

[46] X. Zhao, D. Xu, Y. Li et al., "MicroRNAs regulate bone metabolism," Journal of Bone and Mineral Metabolism, vol. 32, no. 3, pp. 221-231, 2014.

[47] B. Ell and Y. Kang, "MicroRNAs as regulators of bone homeostasis and bone metastasis," BoneKEy Reports, vol. 3, p. 549, 2014.

[48] A. Turchinovich, T. Samatov, A. Tonevitsky, and B. Burwinkel, "Circulating miRNAs: cell-cell communication function?," Frontiers in Genetics, vol. 4, 2013.

[49] A. Turchinovich and W. C. Cho, "The origin, function and diagnostic potential of extracellular microRNA in human body fluids," Frontiers in Genetics, vol. 5, 2014.

[50] B. C. J. van der Eerden, "MicroRNAs in the skeleton: Cellrestricted or potent intercellular communicators?," Archives of Biochemistry and Biophysics, vol. 561, no. 1, pp. 46-55, 2014. 
[51] M. Hackl, U. Heilmeier, S. Weilner, and J. Grillari, "Circulating microRNAs as novel biomarkers for bone diseases - Complex signatures for multifactorial diseases?," Molecular and Cellular Endocrinology, vol. 432, pp. 83-95, 2016.

[52] P. Vrtacnik, J. Marc, and B. Ostanek, "Epigenetic mechanisms in bone," Clinical Chemistry \& Laboratory Medicine, vol. 52, no. 5, pp. 589-608, 2014.

[53] D. Jing, J. Hao, Y. Shen et al., "The role of microRNAs in bone remodeling," International Journal of Oral Science, vol. 7, no. 3, pp. 131-143, 2015.

[54] P. Tang, Q. Xiong, W. Ge, and L. Zhang, "The role of microRNAs in osteoclasts and osteoporosis," RNA Biology, vol. 11, no. 11, pp. 1355-1363, 2014.

[55] A. Bedene, S. Mencej Bedrač, L. Ješe et al., "MiR-148a the epigenetic regulator of bone homeostasis is increased in plasma of osteoporotic postmenopausal women," Wien Klin Wochenschr, vol. 128, no. S7, pp. 519-S526, 2016.

[56] C. Seeliger, K. Karpinski, A. Haug et al., "Five freely circulating miRNAs and bone tissue miRNAs are associated with osteoporotic fractures," Journal of Bone and Mineral Research, vol. 29, no. 8, pp. 1718-1728, 2014.

[57] L. Panach, D. Mifsut, J. Tarín, A. Cano, and M. Á. GarcíaPérez, "Serum circulating microRNas as biomarkers of osteoporotic fracture," Calcified Tissue International, vol. 97, no. 5, pp. 495-505, 2015.

[58] N. Kosaka, H. Iguchi, and T. Ochiya, "Circulating microRNA in body fluid: a new potential biomarker for cancer diagnosis and prognosis," Cancer Science, vol. 101, no. 10, pp. 20872092, 2010.

[59] W. T. Wang, Y. N. Zhao, B. W. Han, S. J. Hong, and Y. Q. Chen, "Circulating microRNAs identified in a genome-wide serum microRNA expression analysis as noninvasive biomarkers for endometriosis," The Journal of Clinical Endocrinology and Metabolism, vol. 98, no. 1, pp. 281-289, 2013.

[60] D. P. Bartel, "MicroRNAs: genomics, biogenesis, mechanism, and function," Cell, vol. 116, no. 2, pp. 281-297, 2004.

[61] H. Li, Z. Wang, Q. Fu, and J. Zhang, "Plasma miRNA levels correlate with sensitivity to bone mineral density in postmenopausal osteoporosis patients," Biomarkers, vol. 19, no. 7, pp. 553-556, 2014.

[62] T. Kranjc, B. Ostanek, and J. Marc, "Bone microRNAs and ageing," Current Pharmaceutical Biotechnology, vol. 18, no. 3, pp. 210-220, 2017.

[63] S. Chen, L. Yang, Q. Jie et al., "MicroRNA-125b suppresses the proliferation and osteogenic differentiation of human bone marrow-derived mesenchymal stem cells," Molecular Medicine Reports, vol. 9, no. 5, pp. 1820-1826, 2014.

[64] J. J. Otten, J. P. Hellwig, and L. D. Meyers, Dietary Reference Intakes: The Essential Guide to Nutrient Requirements, National Academy Press, Washington, DC, 2006.

[65] Q. Wang, P. Nicholson, J. Timonen et al., "Monitoring Bone Growth Using Quantitative Ultrasound in Comparison with DXA and pQCT," Journal of Clinical Densitometry, vol. 11, no. 2, pp. 295-301, 2008.

[66] E. Cadore, M. Izquierdo, M. G. . Santos et al., "Hormonal responses to concurrent strength and endurance training with different exercise orders," Journal of Strength and Conditioning Research, vol. 26, no. 12, pp. 3281-3288, 2012.

[67] S. T. Choi, S. R. Kwon, J. Y. Jung et al., "Prevalence and fracture risk of osteoporosis in patients with rheumatoid arthritis: a multicenter comparative study of the FRAX and WHO criteria," Journal of Clinical Medicine, vol. 7, no. 12, p. 507, 2018.

[68] X. M. Xu, N. Li, K. Li et al., "Discordance in diagnosis of osteoporosis by quantitative computed tomography and dual-energy X-ray absorptiometry in Chinese elderly men," Journal of Orthopaedic Translation, vol. 18, pp. 59-64, 2019.

[69] J. A. Kanis, Melton LJ 3rd, C. Christiansen, C. C. Johnston, and N. Khaltaev, "The diagnosis of osteoporosis," Journal of Bone and Mineral Research, vol. 9, no. 8, pp. 1137-1141, 1994.

[70] A. H. Alghadir, F. A. Aly, and S. A. Gabr, "Effect of moderate aerobic training on bone metabolism indices among Adult Humans," Pakistan Journal of Medical Sciences, vol. 30, no. 4, pp. 840-844, 2014.

[71] S. Gabr, M. Al-Ghadir, A. H. Alghadir, and E. S. Al-Eisa, "Correlation between bone mineral density and serum trace elements in response to supervised aerobic training in older adults," Clinical Interventions in Aging, vol. 11, pp. 265273, 2016.

[72] A. H. Alghadir, S. A. Gabr, and E. S. Al-Eisa, "Mechanical factors and vitamin D deficiency in schoolchildren with low back pain: biochemical and cross-sectional survey analysis," Journal of Pain Research, vol. Volume 10, pp. 855-865, 2017.

[73] A. H. Alghadir, S. A. Gabr, and A. A. Rizk, "Physical fitness, adiposity, and diets as surrogate measures of bone health in schoolchildren: a biochemical and cross-sectional survey analysis," Journal of Clinical Densitometry, vol. 21, no. 3, pp. 406-419, 2018.

[74] B. Gomez Jr., S. Ardakani, J. Ju et al., "Monoclonal antibody assay for measuring bone-specific alkaline phosphatase activity in serum," Clinical Chemistry, vol. 41, no. 11, pp. 1560-1566, 1995.

[75] C. Rosenquist, C. Fledelius, S. Christgau et al., "Serum CrossLaps One Step ELISA. First application of monoclonal antibodies for measurement in serum of bone-related degradation products from C-terminal telopeptides of type I collagen," Clinical Chemistry, vol. 44, no. 11, pp. 22812289, 1998.

[76] T. Hassannia, E. GhaznaviRad, R. Vakili, S. Taheri, and S. A. Rezaee, "High prevalence of vitamin D deficiency and associated risk factors among employed women in a sunny industrial city," International Journal for Vitamin and Nutrition Research, vol. 85, no. 3-4, pp. 119-128, 2015.

[77] A. F. Olubajo, A. O. Adefunke, O. O. Ayinla, and A. Margaret, "Changes in stress index, blood antioxidants and lipid profile between trained and untrained young female adults during treadmill exercise test: a comparative study," Nigerian Journal of Experimental and Clinical Biosciences, vol. 3, no. 1, pp. 1-7, 2015.

[78] H. A. Al-Rawaf, A. H. Alghadir, and S. A. Gabr, "MicroRNAs as biomarkers of pain intensity in patients with chronic fatigue syndrome," Pain Practice, vol. 19, no. 8, pp. 848860, 2019.

[79] F. Safari, H. Hosseini, M. Bayat, and A. Ranjbar, "Synthesis and evaluation of antimicrobial activity, cytotoxic and proapoptotic effects of novel spiro-4H-pyran derivatives," $R S C$ Advances, vol. 9, no. 43, pp. 24843-24851, 2019.

[80] Z. Z. Chen, X. D. Zhang, Y. Chen, and Y. B. Wu, "The role of circulating miR-146a in patients with rheumatoid arthritis treated by Tripterygium wilfordii Hook F," Medicine, vol. 96, no. 20, article e6775, 2017. 
[81] R. Eastell, T. W. O'Neill, L. C. Hofbauer et al., "Postmenopausal osteoporosis," Nature Reviews Disease Primers, vol. 2, no. 1, article 16069, 2016.

[82] L. Oei, F. Koromani, F. Rivadeneira, M. C. Zillikens, and E. H. Oei, "Quantitative imaging methods in osteoporosis," Quantitative Imaging in Medicine and Surgery, vol. 6, no. 6, pp. 680-698, 2016.

[83] M. Mylona, M. Leotsinides, T. Alexandrides, N. Zoumbos, and P. A. Dimopoulos, "Comparison of DXA, QCT and trabecular structure in beta-thalassaemia," European Journal of Haematology, vol. 74, no. 5, pp. 430-437, 2005.

[84] W. Yu, C. Glüer, S. Grampp et al., "Spinal bone mineral assessment in postmenopausal women: a comparison between dual X-ray absorptiometry and quantitative computed tomography," Osteoporosis International, vol. 5, no. 6, pp. 433-439, 1995.

[85] K. Engelke, C. Libanati, Y. Liu et al., "Quantitative computed tomography (QCT) of the forearm using general purpose spiral whole-body CT scanners: Accuracy, precision and comparison with dual- energy X-ray absorptiometry (DXA)," Bone, vol. 45, no. 1, pp. 110-118, 2009.

[86] N. Li, X. M. Li, L. Xu, W. J. Sun, X. G. Cheng, and W. Tian, "Comparison of QCT and DXA: Osteoporosis Detection Rates in Postmenopausal Women," The Journal of Endocrinology, vol. 2013, article 895474, pp. 1-5, 2013.

[87] S. Khosla, L. J. Melton 3rd, and B. L. Riggs, "The unitary model for estrogen deficiency and the pathogenesis of osteoporosis: is a revision needed?," Journal of Bone and Mineral Research, vol. 26, no. 3, pp. 441-451, 2011.

[88] P. Kann, M. Laudes, B. Piepkorn, A. Heintz, and J. Beyer, "Suppressed levels of serum cortisol following high-dose oral dexamethasone administration differ between healthy postmenopausal females and patients with established primary vertebral osteoporosis," Clinical Rheumatology, vol. 20, no. 1, pp. 25-29, 2001.

[89] J. Fan, N. Li, X. Gong, and L. He, "Serum 25-hydroxyvitamin $\mathrm{D}$, bone turnover markers and bone mineral density in postmenopausal women with hip fractures," Clinica Chimica Acta, vol. 477, pp. 135-140, 2018.

[90] J. Zhao, Q. Cai, D. Jiang, L. Wang, S. Chen, and W. Jia, “The associations of serum vitamin $\mathrm{D}$ and bone turnover markers with the type and severity of hip fractures in older women," Clinical Interventions in Aging, vol. Volume 15, no. 15, pp. 1971-1978, 2020.

[91] A. Kharroubi, E. Saba, R. Smoom, K. Bader, and H. Darwish, "Serum 25-hydroxyvitamin D and bone turnover markers in Palestinian postmenopausal osteoporosis and normal women," Archives of Osteoporosis, vol. 12, no. 1, p. 13, 2017.

[92] I. Chiodini and A. Scillitani, "Attuali conoscenze sulla patogenesi dell'osteoporosi: il ruolo dell'increzione di cortisolo [Role of cortisol hypersecretion in the pathogenesis of osteoporosis]," Recenti Progressi in Medicina, vol. 99, no. 6, pp. 309-313, 2008.

[93] P. R. Ebeling, "Vitamin D and bone health: epidemiologic studies," BoneKEy Reports, vol. 3, p. 511, 2014.

[94] D. Bassil, M. Rahme, M. Hoteit, and G. E. H. Fuleihan, "Hypovitaminosis D in the Middle East and North Africa: prevalence, risk factors and impact on outcomes," Dermatoendocrinol, vol. 5, no. 2, pp. 274-298, 2013.

[95] N. A. Morrison, P. M. George, T. Vaughan, M. W. Tilyard, C. M. Frampton, and N. L. Gilchrist, "Vitamin D receptor genotypes influence the success of calcitriol therapy for recurrent vertebral fracture in osteoporosis," Pharmacogenetics and Genomics, vol. 15, no. 2, pp. 127-135, 2005.

[96] D. Feldman and P. J Malloy, "Mutations in the vitamin D receptor and hereditary vitamin D-resistant rickets," BoneKEy Reports, vol. 3, p. 510, 2014.

[97] J. W. Ryan, D. Reinke, M. Kogawa et al., "Novel targets of vitamin D activity in bone: action of the vitamin D receptor in osteoblasts, osteocytes and osteoclasts," Current Drug Targets, vol. 14, no. 14, pp. 1683-1688, 2013.

[98] H. El-Hajj Fuleihan, "Vitamin D deficiency in the Middle East and its health consequences for children and adults," Clinical Reviews in Bone and Mineral Metabolism, vol. 7, no. 1, pp. 77-93, 2009.

[99] B. W. Hollis, "Circulating 25-hydroxyvitamin D levels indicative of vitamin D sufficiency: implications for establishing a new effective dietary intake recommendation for vitamin $\mathrm{D}$," The Journal of Nutrition, vol. 135, no. 2, pp. 317-322, 2005.

[100] A. O. Alsuwadia, Y. M. Farag, A. A. al Sayyari et al., "Prevalence of vitamin D deficiency in Saudi adults," Saudi Medical Journal, vol. 34, no. 8, pp. 814-818, 2013.

[101] T. Shiga, Y. Tsuji, M. Fujioka, and T. Kubo, "Risk factors for hip fracture in Japanese elderly women with osteoporosis: applicability of biochemical markers in bone turnover," Geriatrics \& Gerontology International, vol. 9, no. 1, pp. 69-74, 2009.

[102] Z. Dai, R. Wang, L. W. Ang, J. M. Yuan, and W. P. Koh, "Bone turnover biomarkers and risk of osteoporotic hip fracture in an Asian population," Bone, vol. 83, pp. 171-177, 2016.

[103] R. D. Chapurlat, P. Garnero, G. Brárt, P. J. Meunier, and P. D. Delmas, "Serum type I collagen breakdown product (serum CTX) predicts hip fracture risk in elderly women: The EPIDOS study," Bone, vol. 27, no. 2, pp. 283-286, 2000.

[104] P. Garnero, F. Munoz, E. Sornay-Rendu, and P. D. Delmas, "Associations of vitamin D status with bone mineral density, bone turnover, bone loss and fracture risk in healthy postmenopausal women. The OFELY study," Bone, vol. 40, no. 3, pp. 716-722, 2007.

[105] Z. Akkus, H. Camdeviren, F. Celik, A. Gur, and K. Nas, "Determination of osteoporosis risk factors using a multiple logistic regression model in postmenopausal Turkish women," Saudi Medical Journal, vol. 26, no. 9, pp. 13511359, 2005.

[106] P. M. Furlan, T. ten Have, M. Cary et al., "The role of stressinduced cortisol in the relationship between depression and decreased bone mineral density," Biological Psychiatry, vol. 57, no. 8, pp. 911-917, 2005.

[107] E. of Ekenstam, G. Stålenheim, and R. Hällgren, “The acute effect of high dose corticosteroid treatment on serum osteocalcin," Metabolism, vol. 37, no. 2, pp. 141-144, 1988.

[108] I. Heuser, "The hypothalamic-pituitary-adrenal System in depression," Pharmacopsychiatry, vol. 31, no. 1, pp. 10-13, 1998.

[109] O. Altindag, A. Altindag, M. Asoglu, M. Gunes, N. Soran, and Z. Deveci, "Relation of cortisol levels and bone mineral density among premenopausal women with major depression," International Journal of Clinical Practice, vol. 61, no. 3, pp. 416-420, 2007.

[110] A. K. Freehill and L. G. Lenke, "Severe kyphosis secondary to glucocorticoid- induced osteoporosis in a young adult with 
Cushing's disease. A case report and literature review," Spine, vol. 24, no. 2, pp. 189-193, 1999.

[111] R. M. Reynolds, E. M. Dennison, B. R. Walker et al., "Cortisol secretion and rate of bone loss in a population-based cohort of elderly men and women," Calcified Tissue International, vol. 77, no. 3, pp. 134-138, 2005.

[112] F. Barutta, S. Bellini, R. Mastrocola, G. Bruno, and G. Gruden, "Microrna and microvascular complications of diabetes," International Journal of Endocrinology, vol. 2018, Article ID 6890501, 20 pages, 2018.

[113] Z. Chen, Y. Zhang, C. Liang, L. Chen, G. Zhang, and A. Qian, "Mechanosensitive miRNAs and bone formation," International Journal of Molecular Sciences, vol. 18, no. 8, p. 1684, 2017.

[114] G. E. Grieco, D. Cataldo, E. Ceccarelli et al., "Serum levels of miR-148a and miR-21-5p are increased in type 1 diabetic patients and correlated with markers of bone strength and metabolism," Noncoding RNA, vol. 4, no. 4, p. 37, 2018.

[115] T. Sugatani, J. Vacher, and K. A. Hruska, "A microRNA expression signature of osteoclastogenesis," Blood, vol. 117, no. 13 , pp. $3648-3657,2011$.

[116] L. He and G. J. Hannon, "MicroRNAs: small RNAs with a big role in gene regulation," Nature Reviews Genetics, vol. 5, no. 7, pp. 522-531, 2004.

[117] A. Turchinovich, L. Weiz, A. Langheinz, and B. Burwinkel, "Characterization of extracellular circulating microRNA," Nucleic Acids Research, vol. 39, no. 16, pp. 7223-7233, 2011.

[118] A. Y. Mandourah, L. Ranganath, R. Barraclough et al., "Circulating microRNAs as potential diagnostic biomarkers for osteoporosis," Scientific Reports, vol. 8, no. 1, p. 8421, 2018.

[119] S. Ciuffi, S. Donati, F. Marini, G. Palmini, E. Luzi, and M. L. Brandi, "Circulating MicroRNAs as novel biomarkers for osteoporosis and fragility fracture risk: is there a use in assessment risk?," International Journal of Molecular Sciences, vol. 21, no. 18, p. 6927, 2020.

[120] Y. Shuai, L. Liao, X. Su et al., "Circulating microRNAs in serum as novel biomarkers for osteoporosis: a case-control study," Therapeutic Advances in Musculoskeletal Disease, vol. 12, article 1759720X2095333, 2020. 